\title{
"Chancen für die Bistumszeitungen" \\ Hilfen zur Umsetzung der Erkenntnisse aus der Kommunikatoren- und Rezipientenbefragung und der Inhaltsanalyse
}

von Heiko Klinge

Frau Dr. Renate Köcher, Geschäftsführerin des Instituts für Demoskopie in Allensbach, sagte auf der Herbstvollversammlung der Deutschen Bischofskonferenz 1995 in Fulda in ihrem Bericht den Satz: „Der Glaube oder das Vertrauen an die eigenen Kräfte muß bei den Chefredakteuren und Verlegern wachsen."

Dazu können die Ergebnisse der Feldbefragung und der Inhaltsanalyse ebenso beitragen wie die von der MDG geplanten, angebotenen und durchgeführten Maßnahmen zur Umsetzung dieser Ergebnisse in die Praxis von Verlagen und Redaktionen.

Denn viele Verleger, Redakteure und Vertriebsmitarbeiter sind in ihren beruflichen Erwartungen enttäuscht. Einige Gründe mögen dies stichwortartig belegen:

1. Die Auflagen sinken seit Jahren.

2. In unserer Gesellschaft ist die Position der Kirche schwieriger geworden, und ihre Akzeptanz in der Bevölkerung ist gesunken.

3. Die Stellung der Bistumszeitung innerhalb der Diözese bei Klerus, Hauptamtlichen und Laiengremien könnte besser sein.

4. Die Zukunft der Bistumszeitung, auch aufgrund der kirchlichen Finanzsituation, bereitet Sorge.

Trotz oder wegen dieser oder anderer Gründe gingen die Ergebnisse der Feldbefragungen der Jahre 1975 und 1987 an vielen Bistumszeitungen spurlos vorüber. Auch das gut gemeinte Colloquium "Katholische Presse" mit fast allen Bistumsleitungen in Fürstenried im Jahre 1989 ergab zwar eine Dokumentation, die Ergebnisse wurden jedoch kaum umgesetzt.

Damit es der breit und tief angelegten Analyse 1994/1995 nicht ebenso ergeht, hat die MDG - in Absprache mit der Arbeitsgemeinschaft Katholische Presse (AKP) und der Zentralstelle Medien folgende Umsetzungsmaßnahmen entwickelt, mit denen im Jahre 1995 begonnen wurde.

Heiko Klinge ist Geschäftsführer der Medien-Dienstleistung GmbH, München. 
1. Zweitägige Seminare für alle Mitarbeiterinnen und Mitarbeiter der Verlage und Redaktionen, an denen - im Einzelfall - auch Vertreter der Herausgeber teilnahmen.

Die Institute "Allensbach" und "Mainz" und erfahrene Journalisten aus säkularen Verlagen sind mit den Mitarbeiterinnen und Mitarbeitern die einzelnen Zeitungen kritisch durchgegangen. Dabei wurden Schwachstellen deutlich erkennbar und Verbesserungsvorschläge erarbeitet.

Obwohl nur 18 der 23 Bistumszeitungen an der Feldbefragung teilnahmen, beteiligten sich - bis auf eine - alle Zeitungen an den Seminaren. Über 100 Mitarbeiter begannen damit - so ist zu hoffen mit der Neupositionierung ihres Blattes.

2. Dr. Rüdiger Schulz, Adolf Theobald, Udo Heinze und Hartmut Paeffgen, die Referenten und Moderatoren der Seminare, erstellen aus den Diskussionsbeiträgen der Teilnehmerinnen und Teilnehmer und aus eigenen Erkenntnissen aus der Lektüre aller Bistumszeitungen - im Vergleich mit den Ergebnissen der Feldbefragung - ein sog. "Stylebook" als Anregung für den permanenten Prozeß der Veränderung der Blätter auf den Leser hin.

3. Elf Verlage bzw. Bistümer erteilten bisher der MDG den Auftrag, ihre Bistumszeitung einer Gesamtanalyse $\mathrm{zu}$ unterziehen. Die Unternehmensberater der MDG haben ab 1995 diese Aufgaben unter Zuhilfenahme externer Fachleute - begonnen. Dazu werden die Ergebnisse der Feldbefragung ebenso helfen wie die Daten des AKP/MDG-Betriebsvergleiches.

4. Die ERFA-(Erfahrungsaustausch-)Gruppe der Verleger der Bistumspresse wird sich - von der MDG moderiert - verstärkt mit Benchmarking, dem Vergleich der Daten konkurrierender Unternehmen, $\mathrm{zu}$ befassen haben. Der kollegiale Vergleich und die sich daraus ergebenden Konsequenzen für den eigenen Betrieb sind wichtige Hilfen zur Auflagen-, Erlös- und Kostenverbesserung. Unter dem Motto „vom Besseren lernen“.

5. Die MDG bietet - gemeinsam mit der AKP - Fortbildungsseminare für Verleger, Chefredakteure und Vertriebsleiter an zu den Themen:

- Mitarbeiterorientierte Führung (Herausgeber, Verleger, Redaktionen: ein Team!)

- Marketing als Unternehmensphilosophie

(Das Produkt vom Leser her gestalten: Leser, Redaktion, Verlag)

6. Eine Bistumszeitung entwickelt - moderiert von einem externen Trainer aus dem Zeitschriftenbereich - ein Marketingkonzept. Dieses Konzept wird dann - nach entsprechender Überarbeitung - allen Verlagen angeboten werden. Zur Umsetzung für das eigene Blatt steht auf Wunsch ein Trainer der MDG zur Verfügung. 
7. Ein Personalentwicklungsplan für die Verlagshäuser als Anregung für den Umgang mit Zielvereinbarungen und für eine permanente Fortbildung der Mitarbeiter wird mit einer Personalberatungsfirma erstellt. Die MDG hält den Bereich der Fortbildung - neben der guten und notwendigen Ausbildung durch das Institut zur Förderung Publizistischen Nachwuchses in München - für wichtig.

Für diese personal-, zeit- und finanzaufwendigen Aktivitäten seien nur kurz 3 Begründungen genannt:

a) Die Bistumszeitung muß das Mittel der Kommunikation innerhalb des Bistums und damit zwischen den Gemeinden sein und bleiben. Unter dem Motto „Stärket Eure Brüder!“ und macht sie in schwieriger Zeit "fit für den Dialog mit der Welt" ist sie ein unverzichtbares Binnen-Medium der Kirche.

b) Zur Realisierung des unter a) Genannten sind hohe Anstrengungen von allen Beteiligten gefordert, seien es Herausgeber, Verlagsleiter oder Redakteure.

Zudem ist es unwirtschaftlich, in jedem Bistum für jede Zeitung alle Maßnahmen eigens zu finanzieren. Durch Konzentration der Kräfte ist auch eine höhere Qualität zu erwarten.

c) Die Herausgeber, die Bischöfe, die Bistumsleitungen sind - mit den Verlegern und Chefredakteuren - für die publizistische Effizienz und für ausreichend viele und zufriedene Mitarbeiter verantwortlich. (Daß zufriedene Mitarbeiter wiederum der Effizienz dienen, ist eine Binsenweisheit.) Konsequente Führung, Vertrauen zu den Verantwortlichen, sowie fachliche Kompetenz, Innovationsfreude und Fleiß der Mitarbeiter sind die Selbstheilungskräfte für die anstehenden Probleme.

Neben all den genannten konkreten Maßnahmen sind bestimmte Verhaltensänderungen aller Beteiligten jedoch Voraussetzung für den Erfolg.

Was müßte "nach Allensbach“ und nach allen angebotenen Maßnahmen geschehen?

1. "Nach Allensbach" brauchen die Herausgeber mehr Zeit für das Gespräch mit dem Chefredakteur und dem Verleger, mindestens durch einen autorisierten Vertreter.

2. "Nach Allensbach" sind gemeinsam die Führungsaufgaben wahrzunehmen. (Chefredakteur, Verleger und Herausgeber). Die erkennbare einheitliche Linie ist die Voraussetzung für Klarheit, Verläßlichkeit und Berechenbarkeit für alle Mitarbeiter. Und dies ist eine der wichtigsten Voraussetzungen für gute Arbeit. 
3. "Nach Allensbach" gilt die gemeinsame Sorge den Mitarbeitern in Verlag und Redaktion. Es dürfen - wo nötig - nur hervorragende Mitarbeiter zusätzlich eingestellt werden, und die Qualifizierung vorhandener Mitarbeiter muß gesichert sein.

Das kostet Geld, allerdings investiertes Geld. Wenn an der Verbesserung der Binnenpublizistik des Bistums gespart wird, dann war nicht nur das Geld für die Untersuchung rausgeworfenes Geld, dann sind auch alle künftigen Subventionen zweifelhaft.

4. Die Allensbach-Umfrage ergab auch die Notwendigkeit, die Stellung der Bistumszeitung im Bewußtsein aller Mitarbeiter im Bistum, bei ehrenamtlichen wie bei hauptamtlichen Mitarbeitern, zu stärken.

Daher muß - wo noch nicht vorhanden - ein publizistisches Gesamtkonzept im Bistum die Bistumszeitung zur Mitte haben und auf sie hin ausgerichtet sein. Alle publizistischen Aktivitäten im Bistum sollten in einem publizistischen Gesamtplan abgestimmt und geordnet werden.

5. Es werden sich - neben Verbesserungen und damit Auflagensteigerungen - durch all die Veränderungen auch Einsparungen ergeben. Vor allem wenn jede Bistumszeitung nur das Notwendige "vor Ort" tut, das zwingend im Bistum getan werden muß, anderes jedoch in größeren Regionen gemeinsam geschieht und wieder anderes bundesweit erarbeitet wird: Kooperationen und Fusionen dürfen endlich kein Tabu mehr sein!

Die Bistumszeitungen gehen einen interessanten und hoffnungsvollen Weg. Gehen ihn alle gemeinsam und nicht in jedem Bistum allein, in gegenseitiger Loyalität und Berechenbarkeit, mit großem Fleiß, mutig und bereit zum Wandel, dann gibt es eine "Chance für die Bistumszeitungen“. Denn noch ist die Ausgangslage recht gut. Die verkaufte Auflage liegt bei 1,2 Millionen. Der weiteste Leserkreis liegt bei rund 4,9 Millionen. Der Einsatz und das Vertrauen in die eigenen Kräfte lohnen sich. 